\title{
Test Presentation of Watercolor Pigment of Winsor \& Newton
}

\section{Yaodong Chi}

Department of mechanical engineering, North China electric power University, Baoding 071000, China; chiyaodong1@163.com

Keywords: watercolor, pigment, fine art, Winsor \& Newton.

\begin{abstract}
The essay contains large amounts of experimental test from world-wide artists and chemists, statistics is based on scientific research to illustrate Winsor \& Newton product's performances.
\end{abstract}

\section{Introduction}

With the wild spread of national and international watercolor painting, abundant market is now served in east Asia and European countries. While some of art material merchants may mislabel their products to make consumer confusing and misleading to make unreasonable purchase.

This following essay will illustrate major professional brands and their models available in most countries. The essay contains large amounts of experimental test from world-wide artists and chemists, statistics is based on scientific research without art itself.

Classify:

All watercolor items available could classified to 2 levels: Academic and Artists. From elementary to Advance.

Major companies usually have several series to occupy specific level. However, there is no legal standard to justify a series' performance. Secondly, the quality of pigment is the major concern and single pigment component is obviously better than mixed one in similar level including transparency、

Brightness , solar-resistance (lightfastness) and mix with other colors.

Winsor \& Newton

Artists series

This traditional brand made changes latterly twice, in 2005 and 2009. These changes were based:

1 , better medium ingredients help melt quickly.

2, abandon several worse remarked pre-mixed colors.

3 , release new organic colors

The introduction color guide of Winsor \& Newton is quite trustworthy to notice any poor-resistant color for separate purchase. Their colors are easy dyed into paper and tough to remove. The strength of color is compared to be weak but shares one of the best transparency all around world. (Genuine transparent brand is Utrecht and Daniel \& Smith but not available in Asia).Newly developed colors like quinacridone were added.

Winsor provides a equal quantity of colors in each tune with less selection. The best part is considered to be traditional metal oxide even it is not transparent enough for book-decorating painting. Since this brand is a well-known traditional art material brand despite of modern design, Winsor is more likely to be use for tradition watercolor painting.

Another specific way for Asian artist to use watercolor is in Chinese traditional paper, Which can properly performs the sheen effect without much water. Comparing with traditional Oriental painting material, watercolor provides ultimate transparency and fluctuating capacity to achieve modern style oriental painting. For instance, central academy in Beijing has already begun to use watercolor in Chinese painting courses. Since the consumption level of Chinese artists had developed, more and more materials are available in any places especially by online purchase. On the other hand, material brands are also encouraged to launch new fine or luxury products to stimulate consumption. Japan 
brand JiXiang is also a compatible choice in Asia and it shares a good balance of low transparency and fineness, which could provides sophisticated pattern with traditional texture. But they are no match with European brands about pigment quality and sun-proof ability.

Tube package have $5 \mathrm{ml} 14 \mathrm{ml}$ and $37 \mathrm{ml}$, solid one have half bar ,one bar and a plate triple selection. Smaller package have more selection for rarely seen colors.

Compare with others

Maimeri blu is the professional series of Italian brand Maimeri. The color is resemblance with Winsor's product. And it is quite worthy to buy S1 colors separately(15ml tube). It is a ideal choice for intermediate painter to begin professional series. But abundant quantity of consumers proved that it is not quite transparent as it labeled. The pro of Maimeri is the fine grinding and extremely high saturability. But such satuation is easily to damage by mixing.

Daniel Smith is an newly joined competitor from US, Developed by scientists, Daniel colors is famous for nanometer-level fineness and wide range of single pigment component. Though high concentrate tube is hard to melt again on plate, Daniel Smith is considered to be the best tube watercolor to use in variety of ways to mix and paint. But due to the high trice and large package separately sold without a set, it is exclusive for experienced fine art workers to select .

Old Holand ,a well-known oil color producer since 1664. However, Dedicated pigments not always goes well with watercolor medium and only handcraft tube package is available. Though it is famed by handcraft production and highly concentrated fine pigments, they are not at reasonable price for daily use.

Schimcke Horadam, a Germany brand of oil and watercolor for a long time period. Their professional watercolor Horadam is available after hardened twice officially. And it is also the most stable color to mix together. Usually, there are component difference between solid and tube color. Schmincke did not follow others to aid extra medium to enhance satuation. And the instruction of the products is reliable as a great brand as Winsor. So it is also a choice to purchase separately.

Sennelier is a historical watercolor maker known by limited decorated wooden-cased sets. The best time of joy is considered to be melting without hesitate, Officially due to honey is included. This model shows the major character of formal products -low performance cost ratio and needs to do extra-work to use it properly.

Holbein is totally made in Japan instead of Netherland and the only Asian brand suitable for professional painting. Pure pigments are well grained for four times to make sure the quick melting ability and resolution. Due to Asian tradition of painting in thick paper ,the ability of spread in water is severely limited by excessive textures.

Daler Rewey is a cheap choice for set since 1900 and now pursuits artist level with traditional thick color and exclusive pigments reach antique texture. The fill of color is also way too much to take the place to pigments.

Remberandt is considered to be the best seller in china for intermediate collage students and it is even well packaged than major brands like Winsor and Schimcke. Especially steel solid set is pretty good for use or gifting. Even though a wide range of color is made by mixed pigments, their basic color is quite reliable to use. Separate basic color large tube and basic set is quite worthy.

Blockx is a craft brand from Belgium with the most expensive color per gram. However, handcraft is not so competitive with modern technology and pigments did not fulfil great performance without well graining in auto machine. This would not be a problem for oil color but this makes water color badly rewarded.

Lucas, A hierarchy family brand made in Germany since 1862 and several historical colors like $\mathrm{Pb}$ white. The problem of Lucas watercolor could be excessive organic stuff added and poor quality of natural pigments.

White nights is a series from Russia, which simply packaged color in such low price makes it popular in online purchase. Yet, white nights is not an ideal material to make commercial painting. 


\section{Summary}

Comparing with other brands, Winsor \& Newton is more possible to introduced through cheap class products yet it also provides finest materials. Notice that you should begin your color with set. Separate colors may have extreme characteristic for special work and better try before use. Winsor's academic level is 'Cotman'. Names after a famous England painter, it is widely appeared and highly remarked in varies of sets in any size and material. But it not trustworthy for seldom seen color which made in France.

\section{References}

[1]. Information on: http://www.winsornewton.com/assets/ColourCharts/AWCColChartEng.pdf

[2]. Terry Harrison. Skill of watercolor painting. p. 31-36

[3]. Jianfei Yang. 1500-2000 classical masterpiecies. p. 31-36.

[4]. Information on :https://m.duban.com/note/283259273/ 